\title{
Intervening to Reduce Sedentary Behaviors and Childhood Obesity among School-Age Youth: A Systematic Review of Randomized Trials
}

\author{
May May Leung, Alen Agaronov, Kateryna Grytsenko, and Ming-Chin Yeh \\ School of Public Health at Hunter College, City University of New York, 2180 Third Avenue, New York, NY 10035, USA \\ Correspondence should be addressed to May May Leung, mm.leung@hunter.cuny.edu
}

Received 2 June 2011; Revised 9 August 2011; Accepted 12 August 2011

Academic Editor: Dale Bond

Copyright (C) 2012 May May Leung et al. This is an open access article distributed under the Creative Commons Attribution License, which permits unrestricted use, distribution, and reproduction in any medium, provided the original work is properly cited.

Objective. To assess the effectiveness of interventions that focus on reducing sedentary behavior (SB) among school-age youth and to identify elements associated with interventions' potential for translation into practice settings. Methods. A comprehensive literature search was conducted using 4 databases for peer-reviewed studies published between 1980 and April 2011. Randomized trials, which lasted at least 12 weeks, aimed at decreasing SB among children aged 6 to 19 years were identified. Results. Twelve studies were included; 3 focused only on SB, 1 focused on physical activity (PA), 6 were combined SB and PA interventions, and 2 studies targeted SB, PA, and diet. The majority of the studies were conducted in a school setting, while others were conducted in such settings as clinics, community centers, and libraries. Conclusions. Overall, interventions that focused on decreasing SB were associated with reduction in time spent on SB and/or improvements in anthropometric measurements related to childhood obesity. Several of the studies did consider elements related to the intervention's potential for translation into practice settings.

\section{Introduction}

Childhood obesity has long been recognized as a worldwide growing health concern [1-3]. In the past 2 decades, rates of obesity in the US rose among children aged 6 to 11 years from $11.3 \%$ to $19.6 \%$, as well as from $10.5 \%$ to $18.1 \%$ among adolescents aged 12 to 19 years $[4,5]$. Similarly, Great Britain has experienced a threefold increase of overweight in children between 1984 and 2002 [6], and prevalence of obesity among younger children in China has increased from $1.5 \%$ to $12.6 \%$ between 1989 and 1997 [7]. Early consequences of childhood obesity include asthma, hypertension, and early-onset diabetes mellitus [3]. In addition, childhood obesity has been shown to follow into adulthood [8-11] and may lead to cardiovascular disease, cancer, and an increased chance of mortality after the age of 30 years $[12,13]$.

A majority of previous studies addressing this epidemic have revolved around modifying dietary intake $[14,15]$ and physical activity (PA) [16-18]. However, sedentary behavior (SB) appears to be a lifestyle behavior that is increasingly contributing to the prevalence of childhood obesity [19] as research has shown that obese children are more sedentary than their nonobese counterparts [20]. Sedentary behavior largely consists of media use; however, other behaviors that do not expend significant energy, such as attending classes or playing a musical instrument, have been explored as SB [21-23]. It is estimated that children spend approximately one-third of their waking hours using media, which includes watching TV/videos, playing video games, and personal computing [24]. These SB may in turn displace PA, decrease metabolic rate, and/or serve as a conditioned stimulus for eating [25].

Lifestyle interventions aimed at reducing SB have potential to make an impact; however, limited knowledge exists as to the effectiveness of such interventions. In addition, aspects related to an intervention's potential for translation to practice are important to consider for such a significant public health issue as childhood obesity. The main objective of this paper is to assess the effectiveness of interventions that focus on reducing SB among 
school-age youth. A second objective is to identify the elements of the identified interventions related to potential translation to practice settings, such as cost or health disparity implications and sustainability of intervention impact.

\section{Methods}

2.1. Literature Search. Four databases (Medline, PubMed, PsychInfo, Cochrane Library) were searched for the relevant studies published between 1980 and April 2011. For this paper, such keywords as "sedentary behavior," "sedentary lifestyle," "physical inactivity," "television," "video games," "children," "adolescents," and "intervention" were used alone and/or in combination. Relevant references were extracted and examined, compiling the list in the form of titles and abstracts of the selected studies.

2.2. Inclusion Criteria. Identified studies included those that used an intervention aimed at decreasing SB, separately or in combination with body mass index (BMI) or other anthropometric changes, such as waist circumference or triceps skinfold thickness, among children and adolescents, 6 to 19 years of age. We focused on studies that described randomized trials, conducted in the community, school, home, or clinic setting, which lasted at least 12 weeks, and included such strategies as educational, health promotion, behavioral therapy, counseling, or management strategies at the individual and family levels. Studies whose primary goal was to measure changes in PA levels were included if the change in SB was also measured and specified in the results. Sedentary behavior was defined as media-related behavior (time spent watching TV/videotapes, playing video games), breaks from activity, and activities that do not significantly influence the energy expenditure occurring at rest.

2.3. Exclusion Criteria. Searches were conducted only in the English language. Studies based within a controlled laboratory setting were not considered relevant or generalizable, and therefore, not included in the analysis.

2.4. Selection Process. The results of the preliminary search were reviewed; relevant titles with abstracts were then retrieved. Bibliographies of some systematic review papers were reviewed to identify additional studies. Full articles of relevant abstracts were retrieved for further review. Two authors independently assessed retrieved studies for inclusion based upon the criteria listed above. Any inconsistencies were resolved by discussions with the other author. Summary tables were composed of the selected studies. The tables included study design, setting in which it was conducted, theory, characteristics of the participants, duration of the intervention and followup, brief description of intervention, definition of control group, measures of SB and additional outcomes, key findings, demographic disparities information, and limitations.

\section{Results}

A total of 2.939 abstracts were identified through the initial search process. Upon review, 31 full papers were retrieved for further review by two investigators. Of those 31 papers, 12 studies met the inclusion criteria. Figure 1 outlines the flow of the search process and the number of articles that were identified at each stage of the process.

Three studies $[22,23,26]$ focused only on SB, 1 study was a PA intervention [27], 6 studies [20,21, 28-31] were combined SB and PA interventions, and 2 studies $[32,33]$ targeted SB, PA, and diet. Of the 12 studies, 8 were conducted in the US, 3 in Europe (including the UK, France, and the Netherlands), and 1 in Australia. The majority (7 out of 12) of the studies were conducted in a school setting, while 2 were conducted in a clinic, 1 in community centers, 1 conducted in both community centers and schools, and 1 other was carried out in convenient locations, which included clinics, libraries, and schools.

Table 1 summarizes the study design and characteristics, while Table 2 summarizes the outcome measurements focused on SB and anthropometrics and also key findings of each study. The definition of SB varied across the studies. Listed here are all the forms of SB that were measured: time spent watching TV and videotapes, playing video games, doing homework, reading, listening to music, using a computer, playing a musical instrument, doing artwork or crafts, talking with parents, playing quiet games indoors, and attending classes or club meetings. Due to the diversity in study design, study duration, setting, population, and measurement outcomes of the interventions, a quantitative synthesis of the evidence was not possible. Therefore, a qualitative assessment of the current evidence stratified by targeted behaviors is presented.

3.1. Sedentary Behavior Studies. Three studies $[22,23,26]$ focused on reducing SB in school-aged children. EscobarChaves et al. [26] aimed to reduce TV and other media consumption in families with children of ages 6 to 9 years in Houston, Tex, US. One hundred one families were randomized to either the 6-month intervention, which included a 2-hour workshop and 6 bimonthly newsletters, or a control group. The parents and children also worked together to develop a plan in which alternative activities could be done by the child and family in place of SB. At 6month followup, there was a trend toward reducing media consumption in the intervention group; however, these results were not statistically significant. The intervention did find a positive impact on proxy behaviors hypothesized to lead to media use reductions, which are also recommended by the American Academy of Pediatrics, such as not having a TV in the child's bedroom.

Robinson [22] randomly assigned 3rd and 4th graders in 1 of 2 public elementary schools in San Jose, Calif, US to receive an 18-lesson, 6-month classroom curriculum to reduce TV, videotape, and video game use. The curriculum, which was taught by the regular classroom teachers, included self-monitoring and self-reporting of media use, followed by a TV turnoff, in which children were challenged not to use 


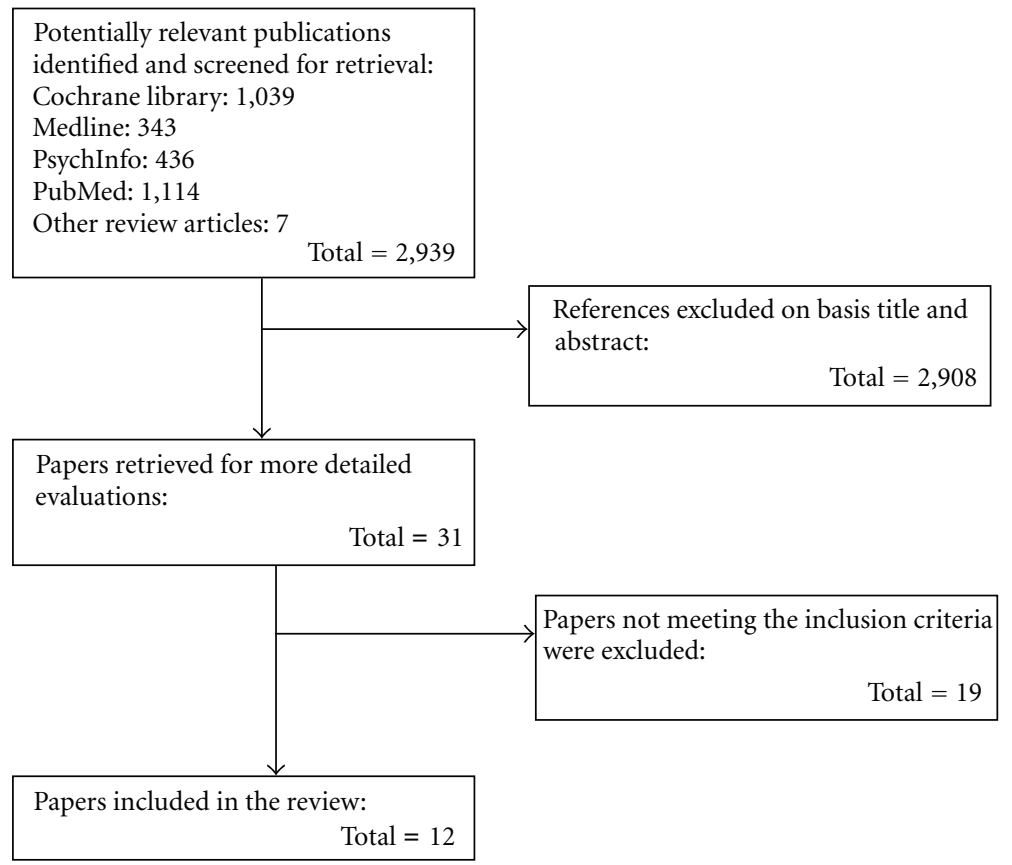

FIGURE 1: Flow chart of the search process.

media for 10 days. After the turnoff challenge, the children were encouraged to follow a 7-hour/week budget of media use. Each household also received an electronic TV time manager, which monitored and bugeted TV/video use for each household member. Newsletters designed to motivate parents to help their children maintain their TV watching limits were also distributed. At the end of the intervention, children in the intervention group had decreases in multiple anthropometric measures, which included BMI, triceps skinfold thickness, waist circumference, and waist to hip ratio $(P<0.002)$, compared to the control group. In addition, reported TV use was lower in the intervention group (8.80 versus 14.46 hours/week; $P<0.001$ ); however, no significant changes were reported in video tape and video game use.

Another study conducted by Robinson and borzekowski [23] consisted of a randomized controlled trial among 3rd and 4th graders in San Jose, Calif, US in 2 public elementary schools $(n=181)$. The intervention was an 18-lesson classroom curriculum focused on reducing screen media exposure. Components of the intervention included children becoming aware of the role TV, videotapes, and video games play in their lives, a TV turnoff in which children attempted to watch no TV/videotapes or play video games for 10 days, children learning how to budget their media use, and participants helping their peers at another school to reduce their media use. Newsletters were also distributed to the parents. Children in the intervention school significantly decreased their weekday TV viewing (1.14 versus 1.96 hours/day; $P<0.001)$ and weekday $(0.19$ versus 0.52 hours/day; $P<0.05)$ and Saturday video game playing $(0.31$ versus 0.9 hours/day; $P<0.05)$ compared to controls. Greater effects were found among boys $(P=0.05)$ and more adult-supervised children $(P=0.03)$.

3.2. Physical Activity Study. One study that focused solely on PA in school-aged children was identified. Slootmaker et al. [27] randomized 87 13-to-17 year olds in Amsterdam, The Netherlands to receive either a single brochure with PA recommendations or an accelerometer and access to webbased tailored PA advice for 3 months. When a user logged into the website and uploaded his/her PA score, the website provided individualized PA feedback based on the current PA score and personally adapted suggestions to promote daily PA. At 5-month followup, time spent doing SB was significantly reduced in boys $(-1,801$ minutes/week; $P=$ 0.04). No SB changes were observed in girls.

3.3. Sedentary Behavior and Physical Activity Studies. Six studies $[20,21,28-31]$ that targeted both SB and PA were identified. Epstein et al. [21] randomized obese children of ages 8 to 12 years from 61 families to 1 of 3 treatment groups: (1) increasing exercise (Exercise), (2) decreasing SB (Sedentary), or (3) both increasing exercise and decreasing SB (Combined). All groups received similar information (distributed through manuals) about the benefits of increased PA and the negative effects of SB; however, the groups differed in the types of activities that were reinforced. The Sedentary group was reinforced for decreasing the amount of time they engaged in certain SB; these SBs included media use, imaginative play, talking on the phone, and playing board games. Participants in the Exercise group were reinforced for increasing PA, while those in the Combined group 


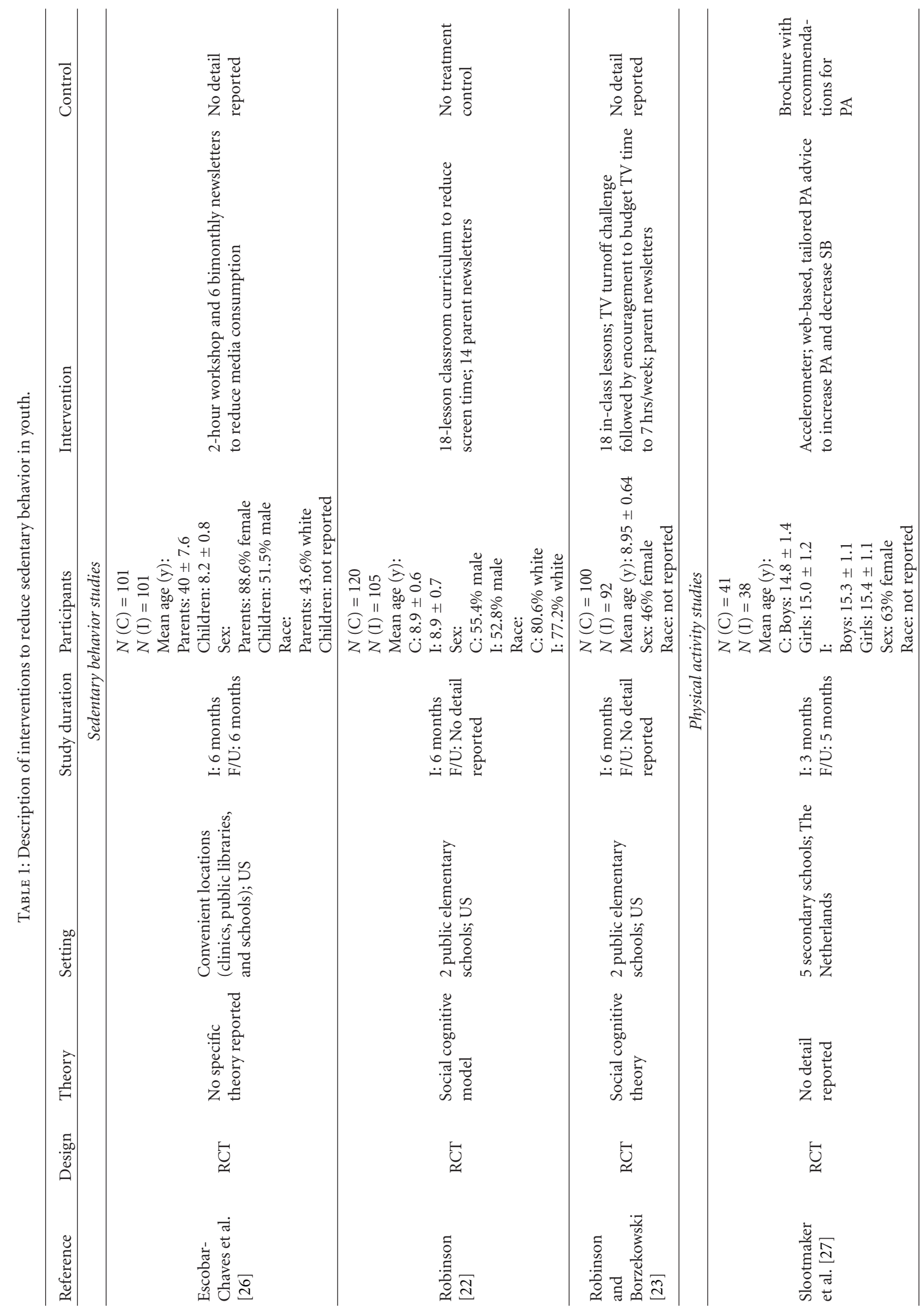




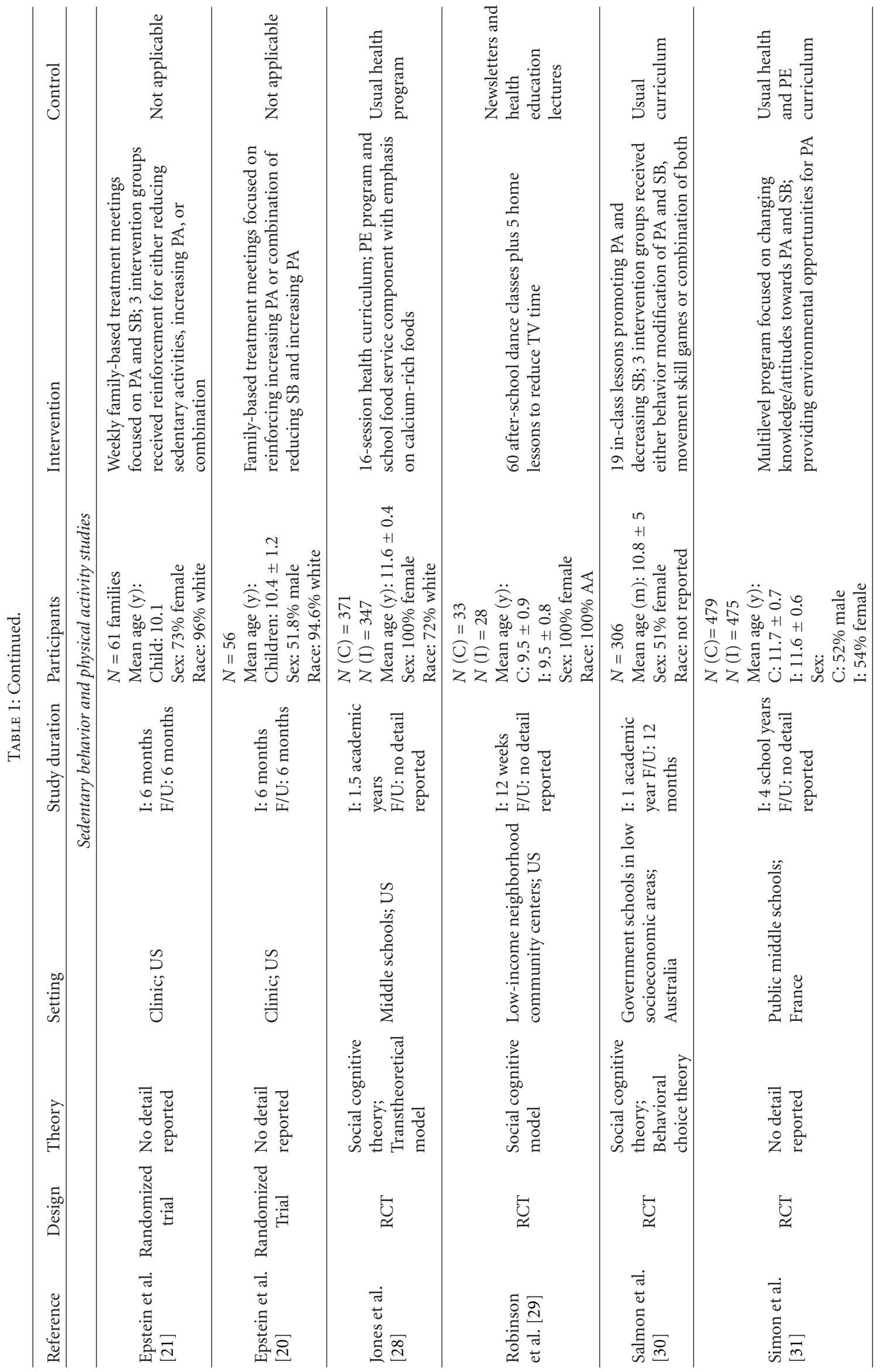




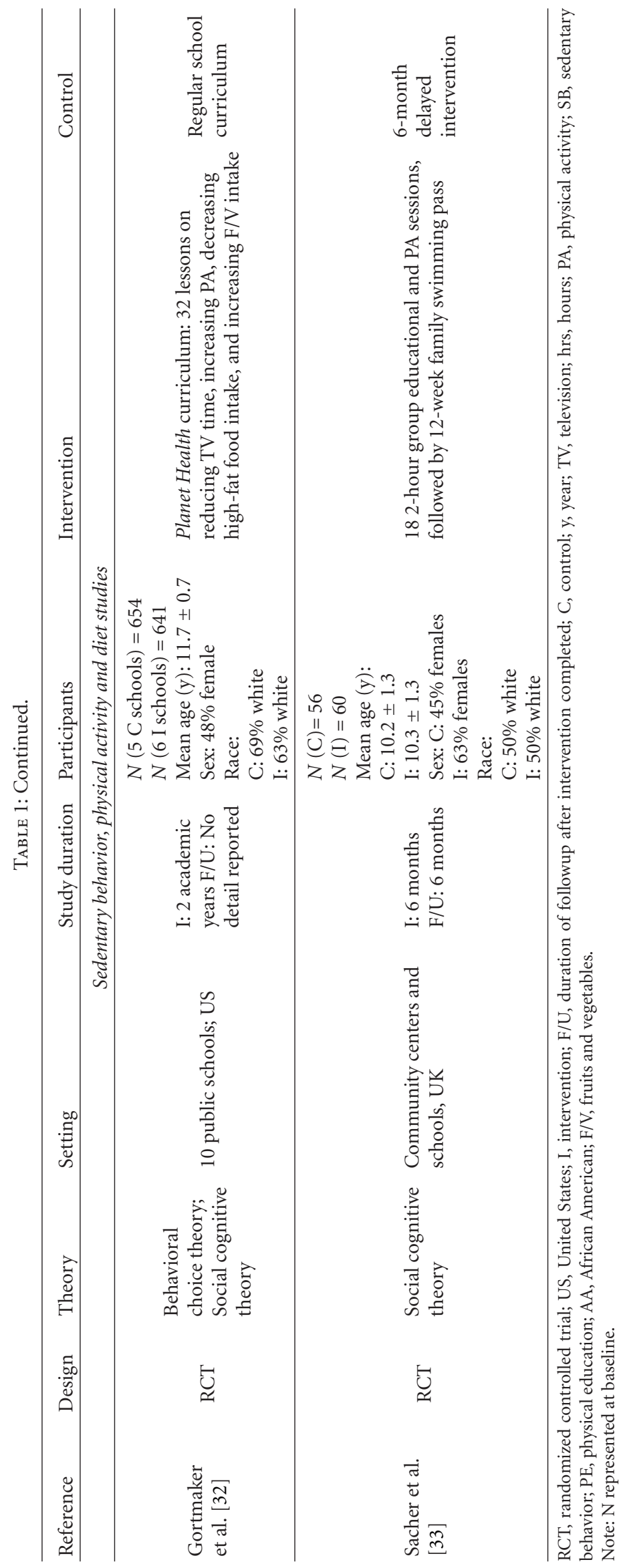




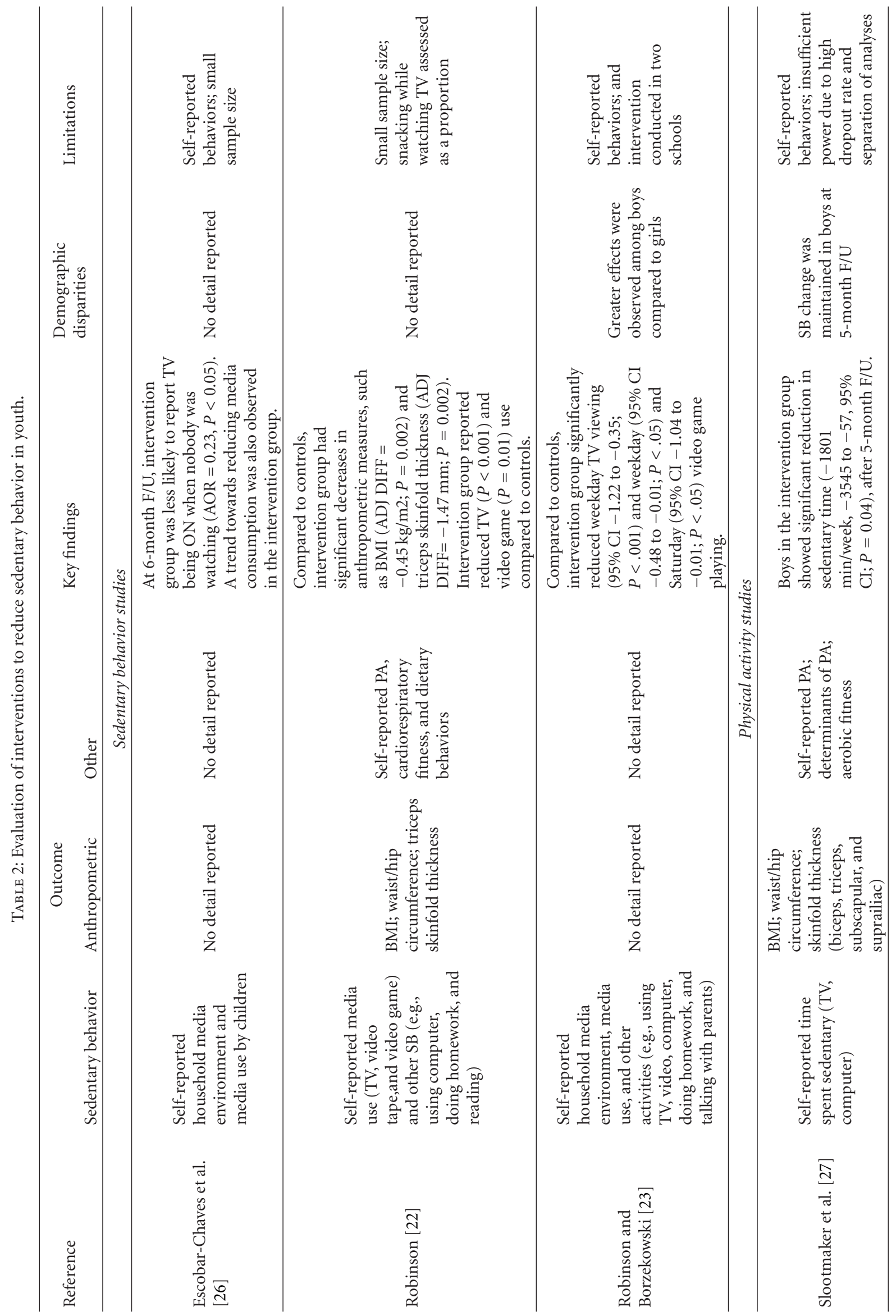




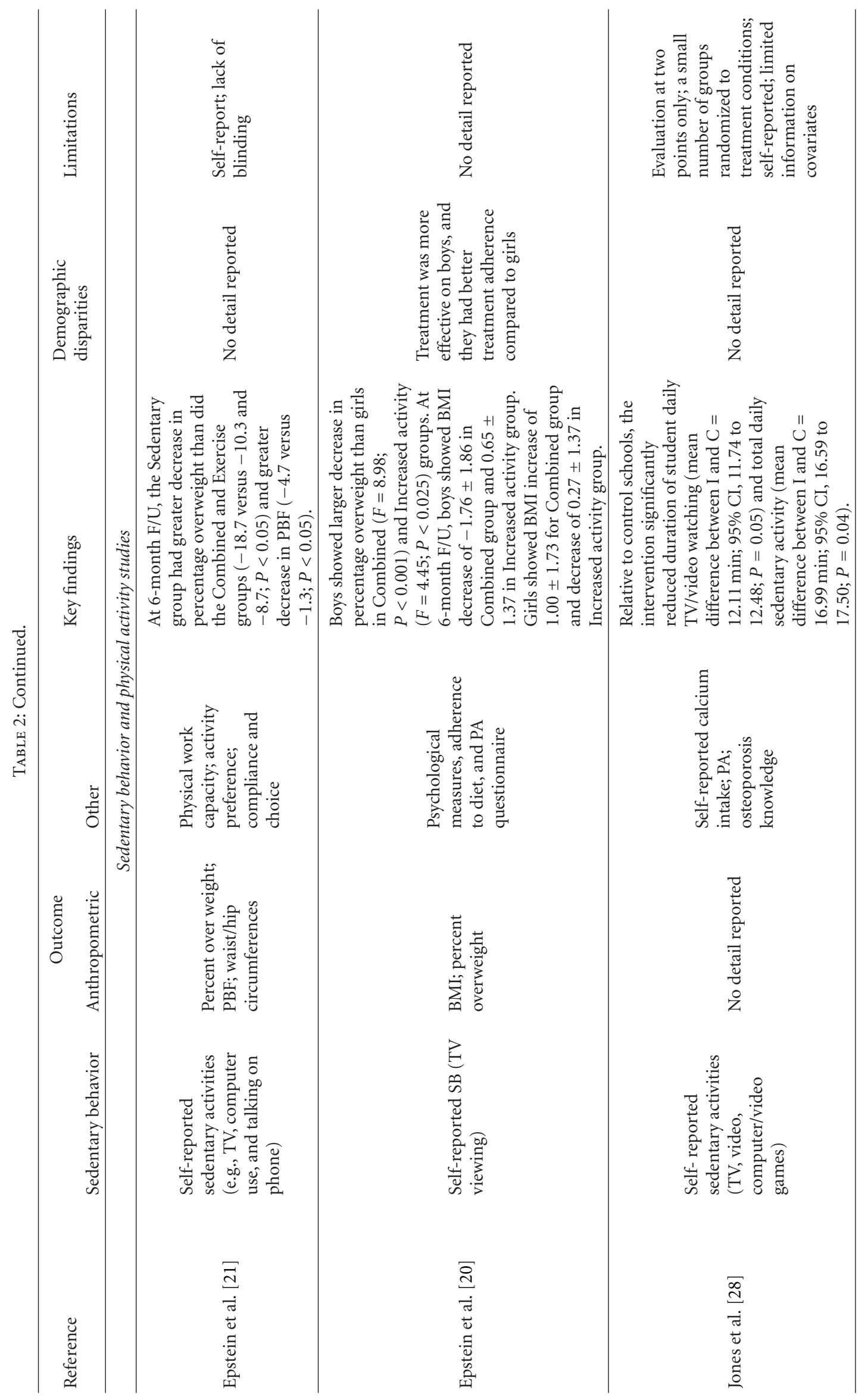




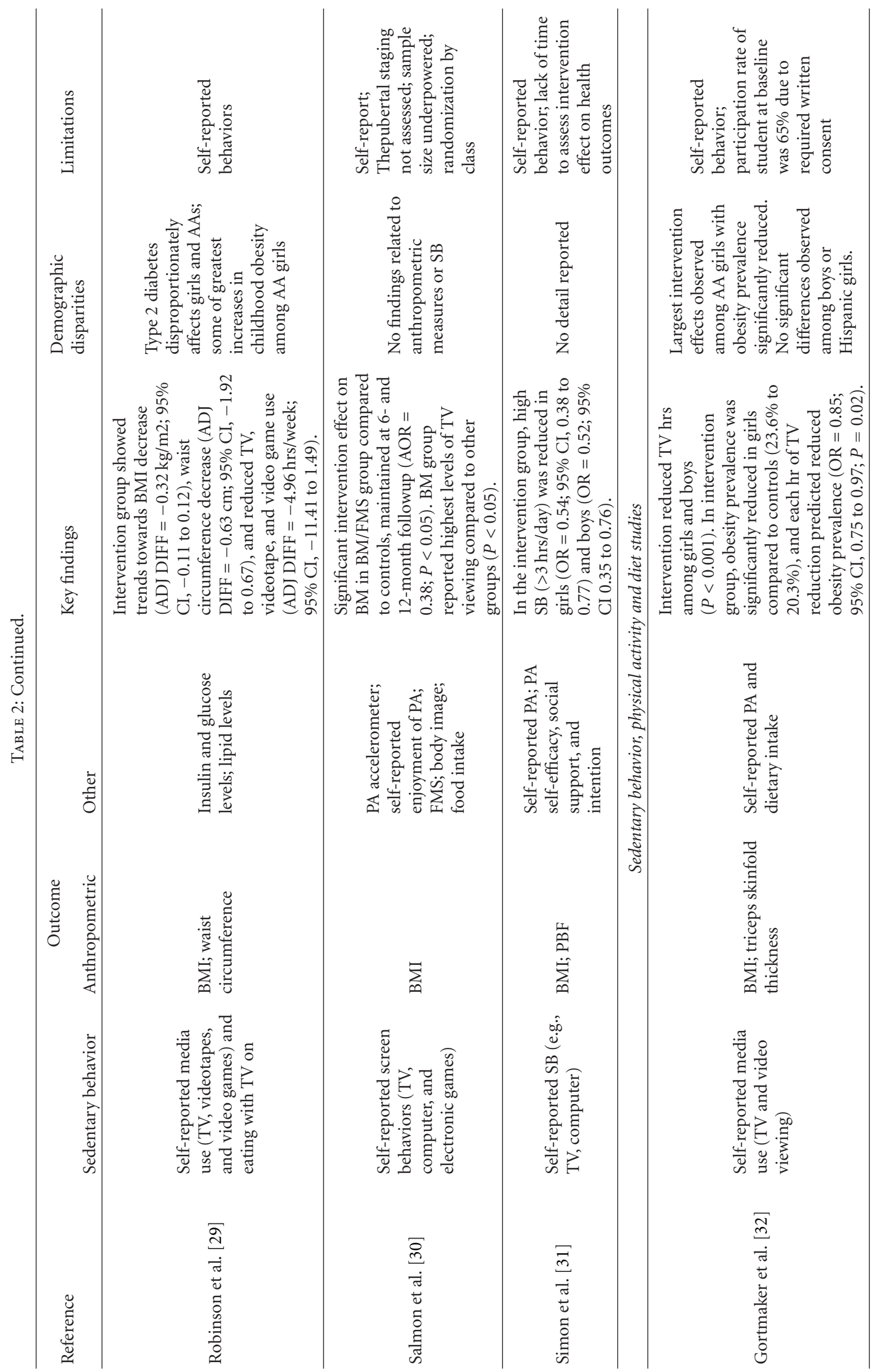




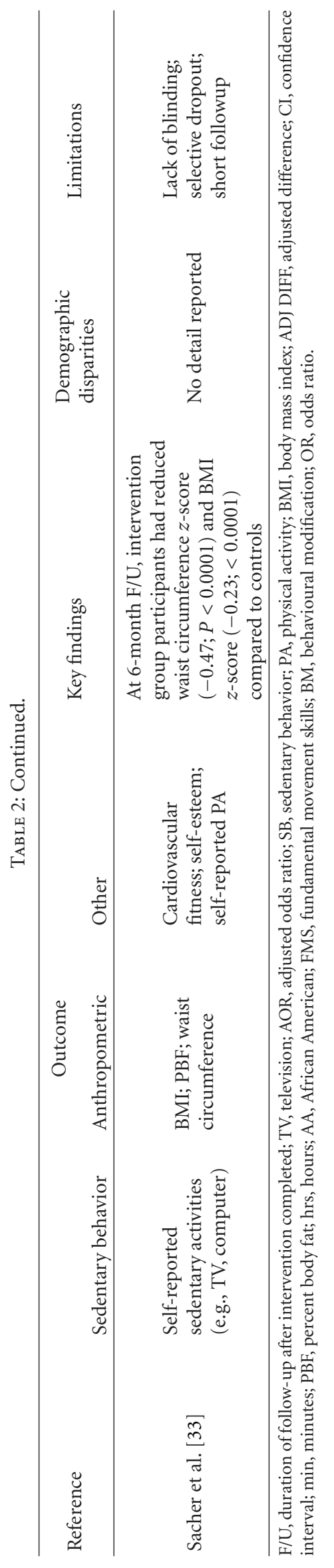


were reinforced for both decreasing SB and increasing PA. Weekly treatment meetings were also conducted for both the parent and child. At 6-month followup, the Sedentary group had greater decrease in percentage overweight than did the Exercise or Combined groups $(-18.7$ versus -10.3 versus $-8.7 ; P=0.026)$ and greater decrease in percentage of body fat $(-4.7$ versus $-1.3 ; P=0.037)$.

Another study by Epstein et al. 2001 [20] randomly assigned 67 families with an obese child between ages of 8 to 12 years to 1 of 2 treatment groups: (1) increasing PA (Increase) or (2) reducing SB and increasing PA (Combined). The treatment program consisted of 16 weekly meetings, followed by 2 biweekly meetings and 2 monthly meetings during a 6-month intensive program. At 6-month followup, boys showed significantly better percentage of overweight changes in the Combined group than girls $(-15.8 \%$ versus $-1.0 \% ; P<0.001)$, with no significant differences in the Increase group for boys or girls $(-9.3 \%$ versus $-7.6 \%)$. Boys also adhered to the treatment better than girls $(P<0.01)$.

Jones et al. [28] recruited 12 middle schools in central Texas to participate in a 1.5-year randomized clinical trial focused on improving bone health mainly through promoting the increase of PA. A total of 718 6th grade girls participated in the intervention, which consisted of a 16-session health curriculum to promote increased weightbearing PA and consumption of calcium-rich foods. A physical education component was also included, which consisted of high-impact activities. Relative to the girls in the control group, the intervention group significantly reduced daily TV and video minutes $(-12.11$ minutes/day; $P=0.05)$. Total daily minutes of sedentary activity were significantly lower for intervention students relative to controls (mean difference between groups $=-17$ minutes; $P=0.04$ ).

Robinson et al. [29] conducted a randomized controlled trial with 61 8-to-10-year-old African-American (AA) girls and their parents. The 12-week intervention consisted of after-school dance classes and a 5-lesson family-based intervention delivered in participants' homes to reduce media use. At followup, the girls in the intervention group had trends towards lower BMI (adjusted difference $=-0.32 \mathrm{~kg} / \mathrm{m}^{2}$; $95 \%$ CI -0.77 to 0.12 ) and waist circumference (adjusted difference $=-0.63 \mathrm{~cm} ; 95 \% \mathrm{CI}-1.92$ to 0.67$)$ and reduced $\mathrm{TV}$, videotape, and video game use (adjusted difference $=$ -4.96 hours/week; $95 \% \mathrm{CI}-11.41$ to 1.49 ).

Salmon et al. [30] randomized, by class, 311 children from 3 government schools in low socioeconomic areas of Melbourne, Australia into one of four conditions: (1) behavioral modification (BM); (2) fundamental movement skills (FMS); (3) combined BM and FMS (BM/FMS); (4) control (usual curriculum). Each of the intervention conditions consisted of 19 lessons promoting PA and decreasing $\mathrm{SB}$. The BM lessons were delivered in the classroom, while the FMS lessons were delivered in PA facilities, which focused on teaching participants physical skills while emphasizing enjoyment and fun. The combined group received both the BM and FMS lessons. There was a significant intervention effect from baseline to postintervention on BMI in the BM/FMS group compared to the control group $\left(-1.88 \mathrm{~kg} / \mathrm{m}^{2} ; P<\right.$ 0.01 ), which was maintained at 6- and 12-month followup $\left(-1.53 \mathrm{~kg} / \mathrm{m}^{2} ; P<0.05\right)$. The BM group reported highest levels of TV viewing compared to the other groups (239.9 minutes/week; $P<0.05$ ).

Simon et al. [31] conducted a 4-year randomized controlled trial with a cohort of 954 middle-school adolescents in eastern France. The multilevel intervention focused on influencing intrapersonal, social, and environmental determinants of PA and SB through informational sessions, social support by parents, peers, teachers, and PA instructors and by providing environmental conditions for PA to encourage students to apply the knowledge and skills they learned. The study is currently on going; thus, data reported here were collected 6 months into the intervention. After 6 months of the intervention, high SB ( $<3$ hours/day) was reduced in both girls and boys $(\mathrm{OR}=0.54$ and $0.52 ; P<0.001)$ in the intervention group compared to the control.

3.4. Sedentary Behavior, Physical Activity, and Diet Studies. Two studies $[32,33]$ focused on modifying SB, PA, and diet. Gortmaker et al. [32] randomized 5 out of 10 middle schools in Massachusetts to receive an interdisciplinary intervention over the course of 2 school years. The intervention, Planet Health, was included in the existing school curriculum of 4 subjects and physical education classes. The sessions focused on decreasing TV viewing, decreasing consumption of high-fat foods, increasing fruit and vegetable intake, and increasing moderate to vigorous PA. Over the 2-year intervention period, obesity prevalence among girls in the intervention schools decreased compared to controls $(\mathrm{OR}=$ $0.47 ; P=0.03$ ), while no differences were observed in boys. The number of hours of TV/video use was reduced in both boys and girls in the intervention group compared to the control group (adjusted difference between groups for boys and girls $=-0.40$ and -0.58 hours/day; $P<0.001$ ).

Sacher et al. [33] recruited 116 obese children in the UK to be randomly assigned to receive the Mind, Exercise, Nutrition, Do it (MEND) program, a multicomponent community-based intervention. This intervention consisted of 18 2-hour group educational and PA sessions held twice weekly in sports centers and schools, in which both parents and children attended. These sessions were followed by a 12week free family swimming pass. At 6 months, participants in the intervention group had a reduced waist circumference $z$-score $(-0.37 ; P<0.0001)$ and BMI $z$-score $(-0.24 ; P<$ $0.0001)$ compared to controls. Significant differences in SB were observed between the intervention and control groups (15.9 versus 21.7 hours/week; $P=0.01$ ). The significant decreases in waist circumference and BMI in the intervention group were sustained up to 9 months after participants completed the educational and PA sessions.

\section{Discussion}

Overall, interventions that focused on decreasing SB, whether alone or in combination with other strategies, such as increasing PA and improving diet, were associated with reduction in time spent on SB and/or improvements in anthropometric measurements related to childhood obesity. 
4.1. Study Design. While the results of the majority of the studies were positive, it is not possible to make any conclusions as to the degree of impact each strategy had on the outcomes due to the variability in study design and outcome measurements. There were only 3 studies $[22,23,26]$ that focused solely on the reduction of SB, and only 1 of those studies [22] collected anthropometric measures. The other 9 studies combined other strategies, such as exercise and healthy eating. Similar results in relation to anthropometric measures and SB were observed in these studies compared to the studies solely focused on reducing SB.

Another aspect of the study designs that made it challenging to interpret any further than a qualitative summary is the variation in how SB was defined. Some studies examined $\mathrm{SB}$ as only media use, while others collected additional measurements, which included behaviors such as talking with parents, playing quiet indoor games, and attending clubs, in addition to media use [21-23]. More consistent measures of different types of SB across studies would assist in determining their relative impact on childhood obesity.

The intensity and dose of the interventions received by participants also varied between interventions. The duration of study periods ranged from 12 weeks to 4 academic years. In addition, some interventions consisted of a workshop and newsletters, while other interventions consisted of multiple lessons and face-to-face encounters with the participants across similar time periods.

Another challenge when assessing impact, particularly when considering potential for translation into practice, was the limited measures of long-term sustainability of the interventions impact. Only 5 out of the 12 identified studies incorporated postintervention follow-up measures [20, 21, $26,27,30,33$ ], which ranged from 5 to 12 months. Overall, a positive long-term impact was observed in either behavioral or anthropometric outcomes in those 5 studies [20, 21, $26,27,30,33]$. However, this highlights the challenges in interpreting impact of the interventions and their potential for translation into real-world settings.

4.2. Common Components. One intervention component that appeared to be repeated in several of the designs was the involvement of family. Whether it was for a clinicbased treatment for obese children or promoting positive behaviors to prevent childhood obesity, parents were engaged to varying degrees. In some interventions, the parents were mailed newsletters to reiterate health messages that were presented to children in school $[22,23]$, while other interventions included having the parent attend workshops/meetings with their children and share in planning healthy events $[20,21,26,29,31,33]$. In one study [23], children who had greater adult supervision were more likely to respond better to the intervention than less supervised children. These study designs and results highlight the importance of having a supportive family environment to promote the positive behaviors that are being targeted.

Another component that was repeated throughout many of the intervention designs was that the children were provided with tangible ideas and appealing alternatives to sedentary activities, and some had the opportunity to choose how to allocate their time [21,26, 29-31]. When children are provided choice among alternative activities, they may perceive increased control over their activity options, so the reduction in SB observed in the studies could be partly explained by the provision of suggestions, ideas, and options for students.

4.3. Demographic Disparities. Four of the studies [20, 23, 27, 32] reported differential effects of the intervention between genders while measuring SB outcomes or anthropometric changes. The impact was inconsistent across the studies. The gender differences were observed in both a family-based weight control treatment [20] and school-based interventions to prevent obesity $[20,23,27,32]$ targeting a range of ages. Two of the studies $[23,27]$ observed a greater effect on boys' SB, while the Epstein et al. [20] study resulted in greater changes in \% overweight in boys compared to girls. On the other hand, the Gortmaker et al. [32] study observed BMI changes in girls, but not boys.

There is no clear explanation as to the differential effects by gender and also why the results were inconsistent across intervention, especially since the interventions were originally designed to reach both males and females. Some suggest that gender differences may vary or become more obvious as children become adolescents, with hormonal and environmental differences between sexes emerging at that challenging stage of development [34-36]; however, gender differences were observed with children as young as 8 years. The differential results may suggest that mediators for $\mathrm{SB}$ or anthropometric changes may be different between males and females; thus, future interventions may need to be tailored specific to gender.

Obesity rates are disproportionate across the ethnicities and socioeconomic status (SES) groups. Reducing such inequalities in childhood obesity is imperative. Some of the studies did address such disparities by either specifically designing interventions to reach certain at-risk populations, such as AA girls or schools in low-SES areas [29,30], or by evaluating results across race/ethnicity or SES groups. However, such study designs and data analysis were limited, warranting further interventions to focus on specifically addressing such inequalities.

4.4. Costs. Understanding the costs related to recruitment and implementation of an intervention and its potential cost effectiveness are important aspects to consider when a health practitioner must determine how best to utilize the oftenlimited resources that are available in community or school settings. In this systematic paper, we aimed to collect any evidence related to cost of the interventions. While there is a need to understand cost-related issues of interventions, unfortunately, as reported in other publications [37], data on cost of the interventions identified for this paper were very limited. Measuring costs related to the different stages of the research process should be incorporated into study designs, and such data should be included when reporting intervention effects. 
4.5. Limitations. There were several limitations to the paper. Similar to other papers, this systematic paper is limited by the quantity and quality of the studies that were identified. A qualitative analysis of the evidence was warranted due to the variations in study design and characteristics, including intervention and follow-up duration, strategies used, population, and measurement outcomes. Measurements of SB were mainly self-report; however, to minimize this potential bias, some studies did use measures with high validity and reliability. In addition, the majority of the studies were conducted in the US, which may limit the generalizability to other countries, where cultural values and behavioral patterns of SB may differ.

\section{Implications}

5.1. For Future Research. This systematic paper highlights the need for future research to further explore the reduction of $\mathrm{SB}$ in relation to preventing and treating childhood obesity. More comprehensive study designs, which include postintervention follow-up measures, are warranted to better understand the impact and potential sustainability of different strategies on outcomes measures related to SB and anthropometry. Additionally, as SB data were mainly self-report, more valid and reliable measures of SB should be developed. Furthermore, addressing childhood obesity inequalities related to race/ethnicity, SES and gender need to be further explored and should be incorporated into the design of future interventions. In addition, a review on cost of the interventions was not possible due to the paucity of available data, thus collecting data related to cost would provide more comprehensive data for public health practitioners to allow them to determine which interventions may be most effective in their settings.

5.2. For Public Health. Many of these interventions, while comprehensive, were designed to be incorporated into the regular school classroom with teachers delivering the lessons. Others were designed to be implemented in convenient locations within communities, and sessions could be led by those without extensive health training or education. One study [27] specifically mentioned that the intervention was designed to make it easily applicable to real-life settings. These study designs point to the important consideration of the often-challenging aspect of feasibility when implementing interventions in real-world settings and highlight interventions that may have a "true public health impact" [38] as behavioral science research must be "contextual" and "practical" [39].

A very limited number of the studies focused on interventions that modified school policies and the physical environment in ways that support improved dietary practices and regular PA. Often such interventions are not candidates for reviews because of their limited outcome measures on specific behaviors or weight-related outcomes. However, such strategies are gaining support and have the potential to make a significant and sustainable impact [40].

In conclusion, interventions aimed at reducing SB appear to be effective in decreasing $S B$ and improvements in anthropometric measures of childhood obesity. In addition, several of the studies did consider elements of feasibility and applicability in real-world settings to increase potential translation of research interventions into practice settings. Childhood obesity is a complex epidemic with various contributing factors at multiple levels. To make an impact on reversing the trends, a combined effort of strategies that address multiple determinants, including SB, across multiple settings, such as the school, community, clinic, and household is needed.

\section{Conflict of Interests}

The authors declare that they have no conflict of interests.

\section{References}

[1] K. Silventoinen, S. Sans, H. Tolonen et al., "Trends in obesity and energy supply in the WHO MONICA Project," International Journal of Obesity, vol. 28, no. 5, pp. 710-718, 2004.

[2] Y. Wang, C. Monteiro, and B. M. Popkin, "Trends of obesity and underweight in older children and adolescents in the United States, Brazil, China, and Russia," American Journal of Clinical Nutrition, vol. 75, no. 6, pp. 971-977, 2002.

[3] C. Gonzalez-Suarez, A. Worley, K. Grimmer-Somers, and V. Dones, "School-based interventions on childhood obesity: a meta-analysis," American Journal of Preventive Medicine, vol. 37, no. 5, pp. 418-427, 2009.

[4] C. L. Ogden, M. D. Carroll, L. R. Curtin, M. M. Lamb, and K. M. Flegal, "Prevalence of high body mass index in US children and adolescents, 2007-2008," JAMA: Journal of the American Medical Association, vol. 303, no. 3, pp. 242-249, 2010.

[5] C. L. Ogden, K. M. Flegal, M. D. Carroll, and C. L. Johnson, "Prevalence and trends in overweight among US children and adolescents, 1999-2000," Journal of the American Medical Association, vol. 288, no. 14, pp. 1728-1732, 2002.

[6] K. L. Rennie and S. A. Jebb, "National prevalence of obesity: prevalence of obesity in Great Britain," Obesity Reviews, vol. 6, no. 1, pp. 11-12, 2005.

[7] J. Luo and F. B. Hu, "Time trends of obesity in pre-school children in China from 1989 to 1997," International Journal of Obesity, vol. 26, no. 4, pp. 553-558, 2002.

[8] S. S. Guo and W. C. Chumlea, "Tracking of body mass index in children in relation to overweight in adulthood," American Journal of Clinical Nutrition, vol. 70, no. 1, pp. 145S-148S, 1999.

[9] M. K. Serdula, D. Ivery, R. J. Coates, D. S. Freedman, D. F. Williamson, and T. Byers, "Do obese children become obese adults? A review of the literature," Preventive Medicine, vol. 22, no. 2, pp. 167-177, 1993.

[10] T. J. Parsons, C. Power, S. Logan, and C. D. Summerbell, "Childhood predictors of adult obesity: a systematic review," International Journal of Obesity, vol. 23, supplement 8, pp. S1S107, 1999.

[11] L. E. Thorpe, D. G. List, T. Marx, L. May, S. D. Helgerson, and T. R. Frieden, "Childhood obesity in New York City elementary school students," American Journal of Public Health, vol. 94, no. 9, pp. 1496-1500, 2004.

[12] National Institutes of Health, "Clinical Guidelines on the identification, evaluation, and treatment of overweight and 
obesity in adults - the evidence report," Obesity Research, vol. 6, supplement 2, pp. 51S-209S, 1998.

[13] V. Burke, L. J. Beilin, D. Dunbar, and M. Kevan, "Associations between blood pressure and overweight defined by new standards for body mass index in childhood," Preventive Medicine, vol. 38, no. 5, pp. 558-564, 2004.

[14] S. Amaro, A. Viggiano, A. Di Costanzo et al., "Kalèdo, a new educational board-game, gives nutritional rudiments and encourages healthy eating in children: a pilot cluster randomized trial," European Journal of Pediatrics, vol. 165, no. 9, pp. 630-635, 2006.

[15] A. S. Ask, S. Hernes, I. Aarek, G. Johannessen, and M. Haugen, "Changes in dietary pattern in 15 year old adolescents following a 4 month dietary intervention with school breakfast-a pilot study," Nutrition Journal, vol. 5, no. 1, article 33, 2006.

[16] A. L. Carrel, R. R. Clark, S. E. Peterson, B. A. Nemeth, J. Sullivan, and D. B. Allen, "Improvement of fitness, body composition, and insulin sensitivity in overweight children in a school-based exercise program: a randomized, controlled study," Archives of Pediatrics and Adolescent Medicine, vol. 159, no. 10, pp. 963-968, 2005.

[17] N. Lazaar, J. Aucouturier, S. Ratel, M. Rance, M. Meyer, and P. Duché, "Effect of physical activity intervention on body composition in young children: influence of body mass index status and gender," Acta Paediatrica, vol. 96, no. 9, pp. 13151320, 2007.

[18] A. L. Liu, X. Q. Hu, G. S. Ma et al., "Report on childhood obesity in China (6) evaluation of a classroom-based physical activity promotion program," Biomedical and Environmental Sciences, vol. 20, no. 1, pp. 19-23, 2007.

[19] J. N. Roemmich, C. M. Gurgol, and L. H. Epstein, "Open-Loop Feedback Increases Physical Activity of Youth," Medicine and Science in Sports and Exercise, vol. 36, no. 4, pp. 668-673, 2004.

[20] L. H. Epstein, R. A. Paluch, and H. A. Raynor, "Sex differences in obese children and siblings in family-based obesity treatment," Obesity Research, vol. 9, no. 12, pp. 746-753, 2001.

[21] L. H. Epstein, A. M. Valoski, L. S. Vara et al., "Effects of decreasing sedentary behavior and increasing activity on weight change in obese children," Health Psychology, vol. 14, no. 2, pp. 109-115, 1995.

[22] T. N. Robinson, "Reducing children's television viewing to prevent obesity: a randomized controlled trial," Journal of the American Medical Association, vol. 282, no. 16, pp. 1561-1567, 1999.

[23] T. N. Robinson and D. L. G. Borzekowski, "Effects of the SMART classroom curriculum to reduce child and family screen time," Journal of Communication, vol. 56, no. 1, pp. 126, 2006.

[24] S. L. Escobar-Chaves and C. A. Anderson, "Media and risky behaviors," Future of Children, vol. 18, no. 1, pp. 147-180, 2008.

[25] L. DeMattia, L. Lemont, and L. Meurer, "Do interventions to limit sedentary behaviours change behaviour and reduce childhood obesity? A critical review of the literature," Obesity Reviews, vol. 8, no. 1, pp. 69-81, 2007.

[26] S. L. Escobar-Chaves, C. M. Markham, R. C. Addy, A. Greisinger, N. G. Murray, and B. Brehm, "The Fun Families study: intervention to reduce children's TV viewing," Obesity, vol. 18, no. 1, pp. S99-S101, 2010.

[27] S. M. Slootmaker, M. J. M. Chinapaw, J. C. Seidell, W. van Mechelen, and A. J. Schuit, "Accelerometers and Internet for physical activity promotion in youth? Feasibility and effectiveness of a minimal intervention [ISRCTN93896459]," Preventive Medicine, vol. 51, no. 1, pp. 31-36, 2010.
[28] D. Jones, D. M. Hoelscher, S. H. Kelder, A. Hergenroeder, and S. V. Sharma, "Increasing physical activity and decreasing sedentary activity in adolescent girls — the Incorporating More Physical Activity and Calcium in Teens (IMPACT) study," International Journal of Behavioral Nutrition and Physical Activity, vol. 5, article 42, 2008.

[29] T. N. Robinson, J. D. Killen, H. C. Kraemer et al., "Dance and reducing television viewing to prevent weight gain in AfricanAmerican girls: the Stanford GEMS pilot study," Ethnicity and Disease, vol. 13, no. 1, pp. S65-S77, 2003.

[30] J. Salmon, K. Ball, C. Hume, M. Booth, and D. Crawford, "Outcomes of a group-randomized trial to prevent excess weight gain, reduce screen behaviours and promote physical activity in 10-year-old children: Switch-Play," International Journal of Obesity, vol. 32, no. 4, pp. 601-612, 2008.

[31] C. Simon, A. Wagner, C. DiVita et al., "Intervention centred on adolescents' physical activity and sedentary behaviour (ICAPS): concept and 6-month results," International Journal of Obesity, vol. 28, supplement 3, pp. S96-S103, 2004.

[32] S. L. Gortmaker, K. Peterson, J. Wiecha et al., "Reducing obesity via a school-based interdisciplinary intervention among youth: Planet Health," Archives of Pediatrics and Adolescent Medicine, vol. 153, no. 4, pp. 409-418, 1999.

[33] P. M. Sacher, M. Kolotourou, P. M. Chadwick et al., "Randomized controlled trial of the MEND program: a family-based community intervention for childhood obesty," Obesity, vol. 18, supplement 1, pp. S62-S68, 2010.

[34] J. N. Roerimich and A. D. Rogol, "Hormonal changes during puberty and their relationship to fat distribution," American Journal of Human Biology, vol. 11, no. 2, pp. 209-224, 1999.

[35] J. Brooks-Gunn and J. A. Graber, "Puberty as a biological and social event: implications for research on pharmacology," Journal of Adolescent Health, vol. 15, no. 8, pp. 663-671, 1994.

[36] M. P. Warren and J. Brooks-Gunn, "Mood and behavior at adolescence: evidence for hormonal factors," Journal of Clinical Endocrinology and Metabolism, vol. 69, no. 1, pp. 7783, 1989.

[37] C. D. Summerbell, E. Waters, L. D. Edmunds, S. Kelly, T. Brown, and K. J. Campbell, "Interventions for preventing obesity in children," Cochrane Database of Systematic Reviews, no. 3, Article ID CD001871, 2005.

[38] P. A. Estabrooks, E. B. Fisher, and L. L. Hayman, "What is needed to reverse the trends in childhood obesity? A call to action," Annals of Behavioral Medicine, vol. 36, no. 3, pp. 209216, 2008.

[39] R. E. Glasgow, "What types of evidence are most needed to advance behavioral medicine?" Annals of Behavioral Medicine, vol. 35, no. 1, pp. 19-25, 2008.

[40] B. Swinburn, "Obesity Prevention in Children and Adolescents," Child and Adolescent Psychiatric Clinics of North America, vol. 18, no. 1, pp. 209-223, 2009. 


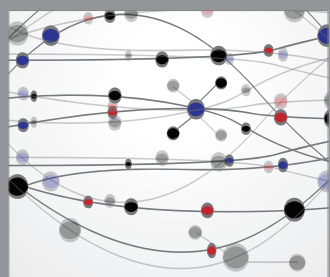

The Scientific World Journal
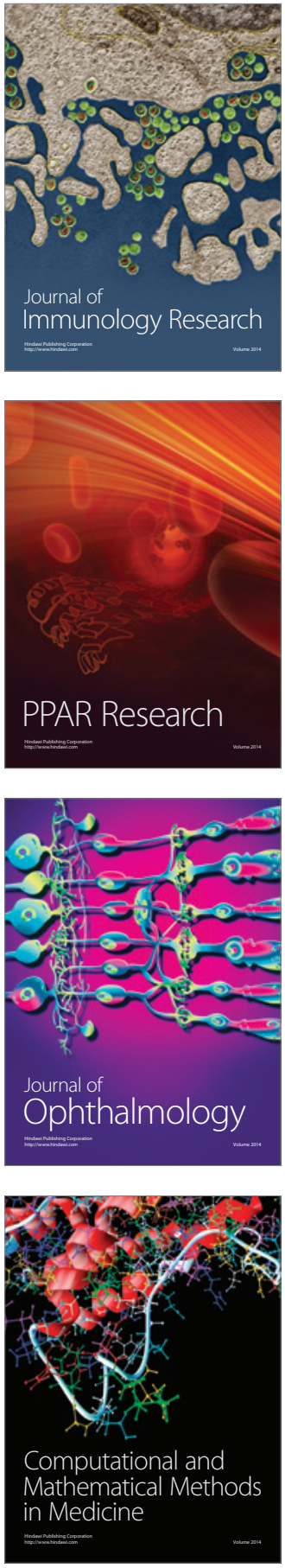

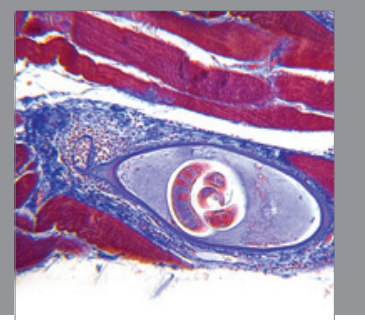

Gastroenterology

Research and Practice
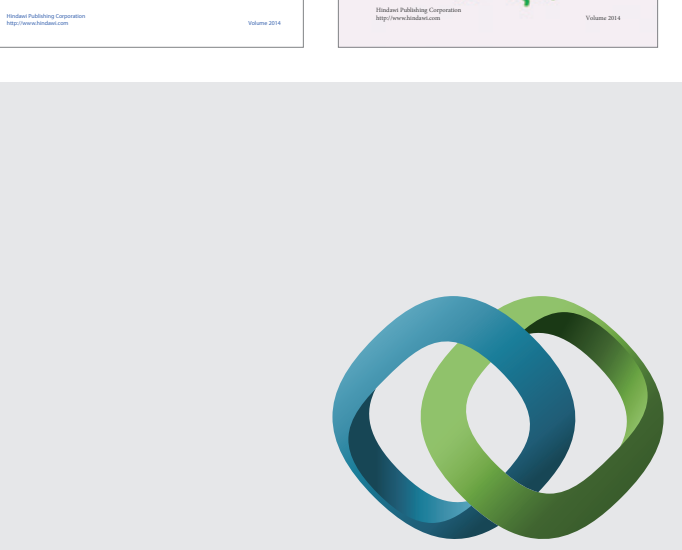

\section{Hindawi}

Submit your manuscripts at

http://www.hindawi.com
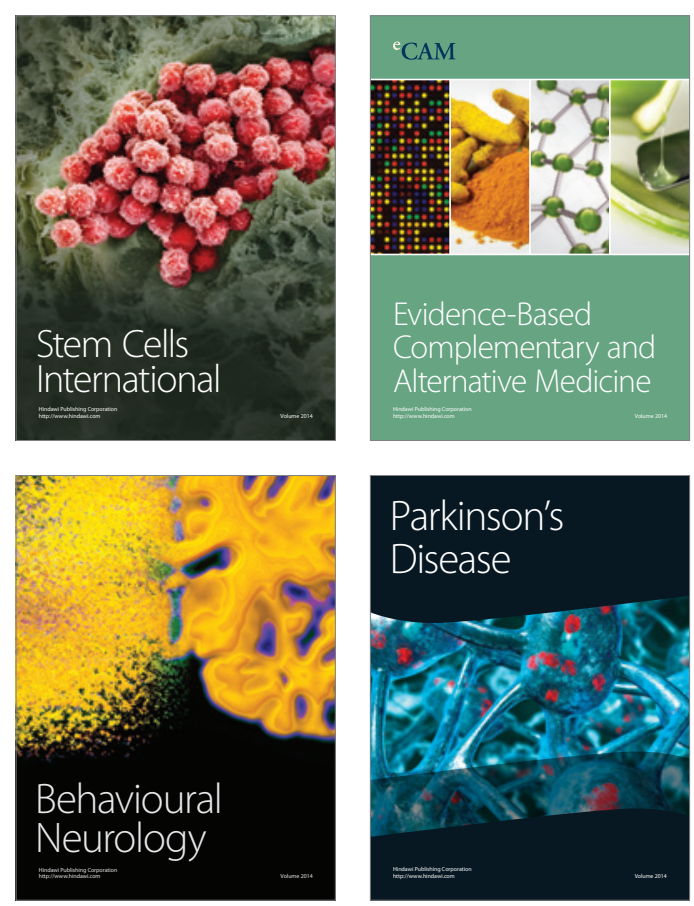

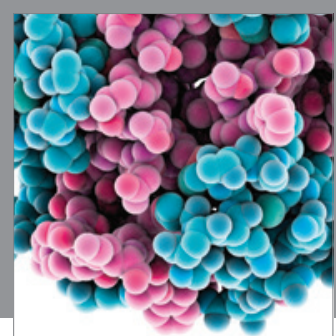

Journal of
Diabetes Research

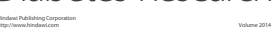

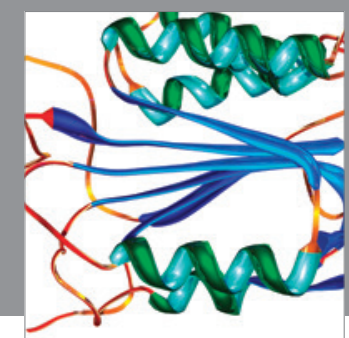

Disease Markers
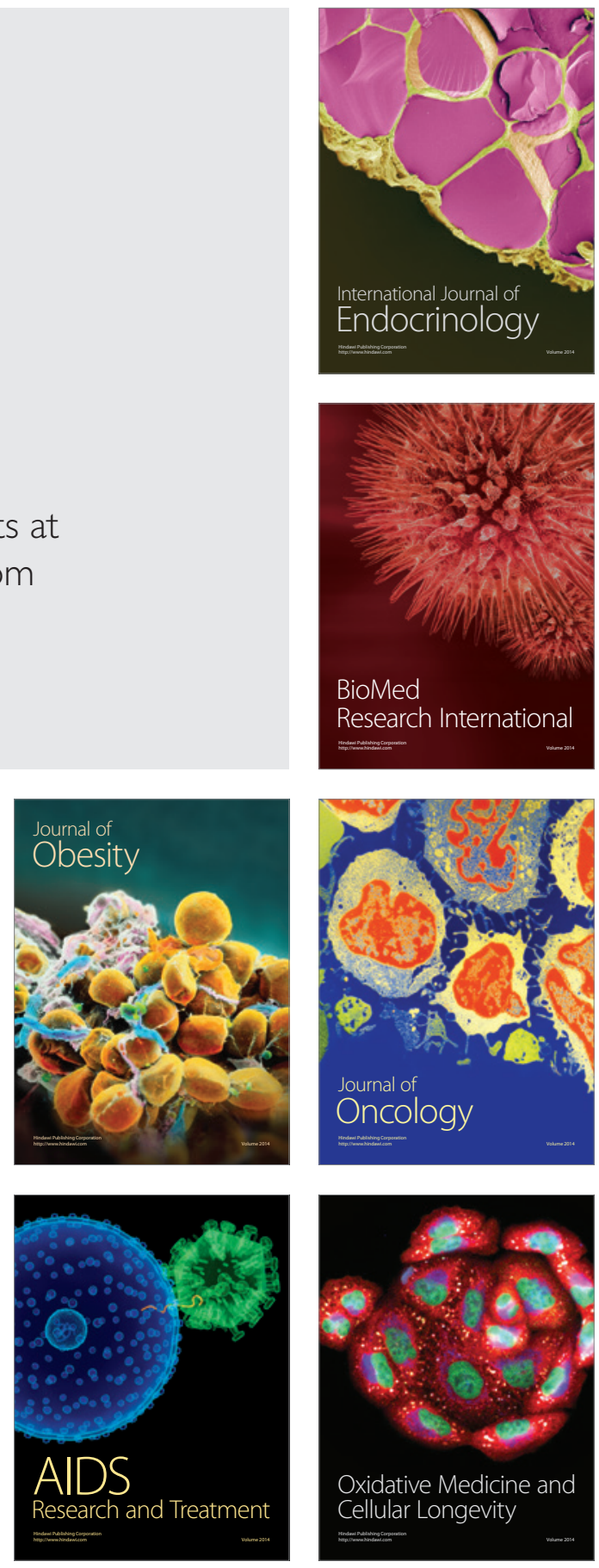